\title{
Parietal Strangulation of Small Intestine in Femoral Hernia Site with Symptoms of Intestinal Obstruction in Patient with Incidentally Found Small Intestine Tumor: A Case Report
}

\section{Andrzej Zyluk ${ }^{\text {** }}$ and Włodzimierz Majewski²}

'Department of General and Hand Surgery, Pomeranian Medical University, Szczecin, Poland

${ }^{2}$ Department of Surgical and Emergency Nursing, Pomeranian Medical University, Szczecin, Poland

\begin{abstract}
Richter's hernia (partial enterocele) is the strangulation/entrapment of only part of the circumference of the intestinal wall. It is relatively rare, and presents without mechanical obstruction - giving vague, non-specific symptoms and signs, and a threat of intestinal necrosis, gangrene, followed by perforation. A report of a case of entrapment of the jejunum in the femoral ring, which did not cause the gangrene, but symptoms of mechanical obstruction, is presented. Further inspection of the small bowel in this case revealed an extra-luminal tumour, which appeared to be a GIST. The entrapped part of the jejunum has been released and the tumour has been resected.
\end{abstract}

Keywords: Intestinal obstruction; Richter's hernia; Strangulation; Small intestine; Tumor

\section{Introduction}

Parietal entrapment of an abdominal hernia (Richter's hernia) is very uncommon. It is a specific version of hernia's strangulation (constriction of the hernial contents by the ring), in which the only a part of the intestinal wall, but not the whole loop is entrapped. Such pathology was described for the first time by Fabricius Hildanus in the $17^{\text {th }}$ century, but first scientific description of parietal intestinal entrapment was presented by August Gottlieb Richter in 1785, and from now on this type of hernia is known by his name [1]. Strangulation usually concerns an intestine wall opposite to the mesentery in a small defect of the abdominal wall, i.e. in femoral, obturator or diaphragmatic hernia [1-3]. These hernias were also reported after laparoscopic operations, occurred in sites of trocar introduction or drains' placement. Parietal entrapment of an abdominal hernia is potentially dangerous, because clinical picture may be unclear, symptoms and signs of mechanical obstruction (typical in strangulated hernias) may be not present, but necrosis and gangrene of the strangulated bowel may secretly develop, leading to perforation and peritonitis $[1,-3]$.

This paper presents a case of entrapment of the jejunum in the femoral ring, which did not cause the gangrene, but symptoms of mechanical obstruction. Over and above, further inspection of the small intestine revealed an extra-luminal tumour, which appeared to be a gastro-intestinal stromal tumor (GIST).

\section{Case Report}

A 71-year old man was admitted to the gastroenterology department, with a diagnosis of obstruction to the small intestine, confirmed by contrast medium radiology. He was admitted to the gastroenterology department for rectal polypectomy, however, the procedure was postponed because of severe pneumonia. On the $9^{\text {th }}$ day of successful pneumonia treatment, clinical symptoms of intestinal obstruction appeared, i.e., mild pain of the abdomen, moderate distension, stop of faeces and flatus for 2 days and two episodes of vomiting. A plain abdominal X-ray revealed fluid levels in small intestinal loops distended up to $5 \mathrm{~cm}$, which suggested intestinal obstruction. There was no evidence of any laparotomy in the patient's history, but he underwent right inguinal hernia operation by Lichtenstein method one year before. The character of pain was colic, of mild intensity, the abdomen was slightly distended and tender at palpation. Examination of inguinal regions performed by an experienced surgeon (consultant) did not reveal any pathology such as inguinal or femoral hernia. An aspiration form the nasogastric tube gave small amount of congestive gastric fluid. Because the patient's shape was relatively good and clinical presentation not evident, a barium meal was administered to distinguish if the obstruction is complete (absolute) or incomplete (partial). This is a standard procedure in doubtful cases of mechanical obstruction performed in our institution. An abdominal X-ray performed 16 hours after commencement of the barium meal showed stop of contrast passage in the distal part of the jejunum (Figure 1). This result confirmed absoluteness of the obstruction and necessity of the operation.

After short preparing of the patient (fluid and electrolyte replacement), the abdomen was opened by lower median laparotomy. Small amount (about $100 \mathrm{ml}$ ) of light fluid was present in the peritoneal cavity, the small intestine was moderately distended and its distal part was found to be fixed in the right minor pelvic region. Further inspection revealed that the bowel is entrapped in the internal ring of the femoral hernia, below the deep inguinal ring. An attempt of retrieving of the bowel by traction failed, thus, a preperitoneal space was prepared what enabled release of the intestine by gentle manipulation, "en block" with hernial sac. The entrapped fragment of the wall of the small intestine was large enough to produce mechanical obstruction. As the released intestine wall looked alive (Figure 2) we decided to leave it without any intervention. The diameter of the orifice of the femoral hernia was relatively small, (about $1.5 \mathrm{~cm}$ ), thus it was simply closed "from inside" with three, non-absorbable sutures.

Inspection of the small intestine revealed a surprisingly large $(7 \times 4$ $\mathrm{cm}$ ) fusiform tumour, growing out of the ileum, at a distance of 1 meter from Treitz's ligament (Figure 3). The structure of the tumour

*Corresponding author: Zyluk A, MD, PhD, Department of General and Hand Surgery, Pomeranian Medical University, Szczecin, Poland, Tel: +48 692968 336; E-mail: :azyluk@hotmail.com

Received February 15, 2015; Accepted March 14, 2015; Published March 21 2015

Citation: Zyluk A, Majewski W (2015) Parietal Strangulation of Small Intestine in Femoral Hernia Site with Symptoms of Intestinal Obstruction in Patient with Incidentally Found Small Intestine Tumor: A Case Report. Surgery Curr Res 5 : 225. doi:10.4172/2161-1076.1000225

Copyright: @ 2015 Zyluk A, et al. This is an open-access article distributed under the terms of the Creative Commons Attribution License, which permits unrestricted use, distribution, and reproduction in any medium, provided the original author and source are credited. 


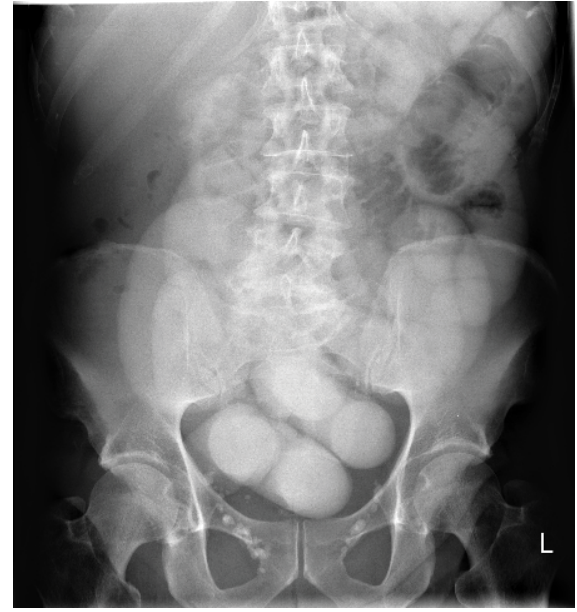

Figure 1: Plain abdominal X-ray 16 hours after starting a barium meal Stop of the passage at the terminal jejunum, suggesting complete mechanical obstruction.

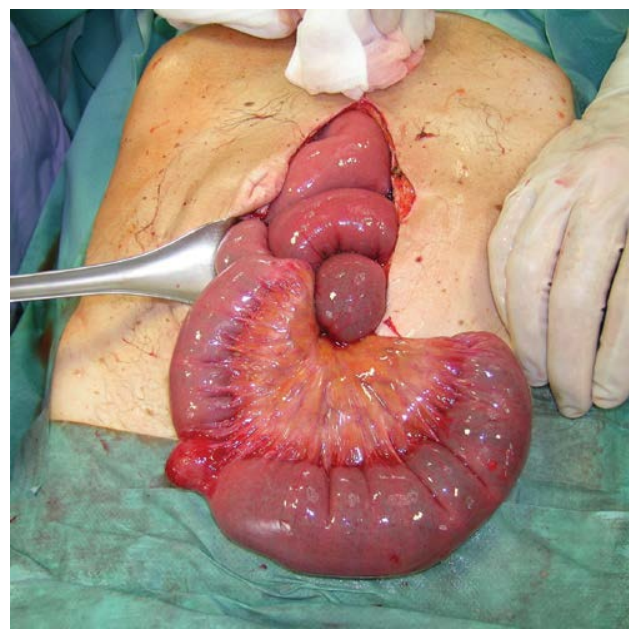

Figure 2: Entrapped and strangulated fragment of the jejunum,released form the orifice of the femoral hernia.

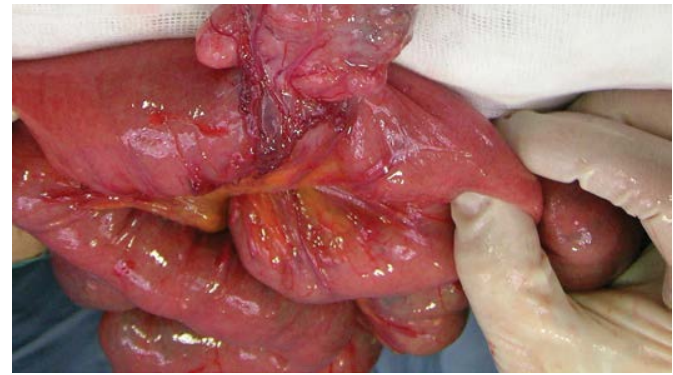

Figure 3: Tumor of the small bowel revealed accidentally during control of the small bowel after release of the strangulated jejunum.

was solid without features of inflammation and was not looking like inflamed Meckel's diverticulum. Because the attachment of the tumour only partly encompassed the intestine it was resected with an adequate margin by linear stapler. The postoperative course was uneventful and the patient was discharged home in sixth postoperative day. A control chest X-ray revealed withdrawal of pneumonia signs and the patient was placed on the list for polypectomy. Histological examination of the lesion revealed a stromal tumour (GIST) of uncertain malignancy (lack of atypia, less than 5/50mitotic figures), therefore requiring no additional treatment.

\section{Discussion}

The presented case seems interesting for the extremely rare cause of intestinal obstruction, as it was parietal strangulation of the intestinal wall at the femoral hernia site without any symptoms in the groin-femoral region. Poorly-symptomatic course in femoral hernia strangulations have been described in the literature but it usually concerned obese, elderly women $[2,3]$. Our patient was rather slim $(\mathrm{BMI}=23)$, the groin region was easily palpable and the examination revealed no pathology. However, the entrapped jejunum fragment was large enough to produce complete mechanical obstruction. Our intraoperative observations suggest that it was not a "classic" acute strangulation of the bowel in the femoral hernial ring, but rather its adhesion to the hernial sac, occurring earlier as a consequence of sticking in the shallow hernial sac. Thus, it was rather irreducible (incarcerated) hernia, which was complicated by mechanical obstruction, because of slowly progressing stricture of the hernial orifice. This supposition confirms the fact, that reduction of the hernia was not possible by simply pulling the bowel, but it required preperitoneal preparation and release "en block" with the hernial sac. Next argument is that the entrapped fragment of intestine was not ischemic. This supports the scenario that the entrapment occurred earlier, with subsequent adhesion of the intestine to the hernial sac, and this resulting in intestinal obstruction.

With regard to diagnostics of the presented case, the clinical symptoms and signs in this patient were not clear, suggesting rather incomplete (not absolute) mechanical obstruction and this was the reason to start with barium meal, which is a good diagnostic test to differentiate absolute from partial mechanical obstruction. Patient's general status was good, therefore postponing of the decision about operation seemed to be not risky. Using of abdominal CT scanning with contrast would be probably better option in this case, but a barium meal is routinely administered as an institutional rule. There is, however, an increasing tendency to use CT scanning in unclear abdominal cases.

We found in the literature some reports on parietal entrapment of the bowel in various defects of the abdominal wall, i.e. femoral, obturator and diaphragmatic hernias' rings, sacral opening and in sites of trocar introduction during laparoscopic surgery or drains placement [1-6]. This type of entrapment usually concerns elderly patients, women more frequently than men, and entrapment usually affects the jejunum [1]. Symptoms of intestinal obstruction develop in some of these patients, facilitating the diagnosis and decision making. "Pure" parietal entrapment, without bowel obstruction, is challenging because of unclear clinical course and frequently the proper diagnosis is made when symptoms signs of peritonitis will appear, caused by the necrosis and perforation of the intestine [2,3,5]. Reports from the literature show abdominal CT scanning to be useful in diagnosing cases of parietal strangulation manifesting with poorly localized abdominal pain, without signs of obstruction [3,5]. In cases with fixed diagnosis of obstruction or perforation and in doubts, surgery is recommended by laparotomy or laparoscopy, which enables revealing and repair the pathology $[1,2,7]$.

Stromal tumors localized in the small intestine are rare, more growing outside and not into the lumen of the intestine. In the literature there are case descriptions of in growing GIST tumors causing intestinal obstruction or bleeding [8,9]. Tumors can occur in any part of the small intestine, and externally can grow large, being asymptomatic. 
Citation: Zyluk A, Majewski W (2015) Parietal Strangulation of Small Intestine in Femoral Hernia Site with Symptoms of Intestinal Obstruction in Patient with Incidentally Found Small Intestine Tumor: A Case Report.Surgery Curr Res 5: 225. doi:10.4172/2161-1076.1000225

The recommended treatment comprises tumor removal with margin of the healthy intestinal wall and anastomosis. GIST tumors can have a different degree of malignancy, depending on tumor size and cell differentiation. The so-called mitotic index is described in every histologic investigation. In advanced GIST treatment even if liver metastases are present chemotherapy with tyrosine kinase inhibitor imatinibe (Glyvec) can be relatively effective.

\section{References}

1. Skandalakis PN, Zoras O, Skandalakis JE, Mirilas $P(2006)$ Richter hernia: surgical anatomy and technique of repair. Am Surg 72:180-4.

2. Vervest AM, Eeftinck Schattenkerk M, Rietberg M (1998) Richter's femoral hernia: a clinical pitfall. Acta Chir Belg 98: 87-99.

3. Mohammad SK (2005) Obturator hernia of the Richter type, a diagnostic challenge. Ugeskr Laeger 167: 3312-3313.
4. Williams MD, Flowers SS, Fenoglio ME, Brown TR (1995) Richter hernia: a rare complication of laparoscopy. Surg Laparosc Endosc 5: 419-421.

5. Matthews BD, Heniford BT, Sing RF (2001) Preperitoneal Richter hernia after a laparoscopic gastric bypass. Surg Laparosc Endosc Percutan Tech 11: 47-49.

6. Botsford TW (1977) Richter hernia in a sacral foramen: new site for richter hernia. Arch Surg 112: 304-305.

7. Ginesta C, Saavedra-Perez D, Valentini M, Vidal O, Benarroch G, et al. (2013) Total extraperitoneal (TEP) hernioplasty with intestinal resection assisted by laparoscopy for a strangulated Richter femoral hernia. Surg Laparosc Endosc Percutan Tech 23: 334-336.

8. Atmatzidis $\mathrm{S}$, Chatzimavroudis $\mathrm{G}$, Ananiadis $\mathrm{A}$, Kapoulas $\mathrm{S}$, Atmatzidis $\mathrm{K}$ (2013) Giant GIST of the small intestine in a young man. J Gastrointest Surg 17: $1536-1537$.

9. Misawa S, Takeda M, Sakamoto H, Kirii Y, Ota H, et al. (2014) Spontaneous rupture of a giant gastrointestinal stromal tumor of the jejunum: a case report and literature review. World J Surg Oncol 12: 153. 